\title{
Adequação do suporte nutricional na terapia nutricional enteral: comparação em dois hospitais ${ }^{1}$
}

\author{
Adequacy of nutritional support provided by \\ enteral feeding: a comparison of two hospitals
}

Vanessa Taís NOZAKI ${ }^{2}$

Rosane Marina PERALTA

RE S U M O

Objetivo

Avaliar o estado nutricional de pacientes hospitalizados e comparar as condutas nutricionais enterais adotadas em dois hospitais gerais da região metropolitana de Maringá (PR), Brasil.

\section{Métodos}

O estudo foi realizado com 62 pacientes de ambos os sexos, em uso apenas de nutrição enteral. Os dados foram coletados em dois hospitais gerais. As necessidades energéticas dos pacientes foram calculadas por meio das equações propostas por Harris \& Benedict e pela recomendação da European Society for Clinical Nutrition and Metabolism. O estado nutricional dos pacientes foi avaliado utilizando-se medidas antropométricas.

\section{Resultados}

Altos índices de desnutrição foram encontrados em ambos os hospitais, especialmente avaliando-se a Área Muscular do Braço. A prescrição energética mostrou-se adequada para 45,71\% e 40,74\% dos pacientes dos hospitais 1 e 2, respectivamente.

\section{Conclusão}

Inadequações na terapia nutricional enteral, associadas a elevados índices de desnutrição, foram detectadas em ambos os hospitais. Os dados obtidos neste estudo demonstram a necessidade de melhoras nos dois serviços de nutrição enteral, sendo necessária a adoção de medidas de padronização e avaliação periódica dos pacientes.

Termos de indexação: Estado nutricional. Nutrição enteral. Terapia nutricional.

\footnotetext{
1 Artigo elaborado a partir da dissertação de V.T. NOZAKI, intitulada "Adequação do suporte nutricional na terapia nutricional enteral em dois hospitais da microrregião de Maringá, PR". Universidade Estadual de Maringá; 2007.

2 Universidade Estadual de Maringá, Centro de Ciências da Saúde, Programa de Pós-Graduação em Ciências da Saúde. Av. Colombo, 5790, 87020-900, Maringá, PR, Brasil. Correspondência para/Correspondence to: V.T. NOZAKI. Email: <vanessa.tais@bol.com.br>
} 


\section{A B S T R A C T}

\section{Objective}

The objective of this study was to assess the nutritional status of inpatients and compare the enteral feeding practices of two general hospitals in the metropolitan region of Maringá (PR), Brazil.

\section{Methods}

The study included 62 patients of both sexes on exclusive enteral feeding. The data were collected in two general hospitals. The energy requirements of the patients were estimated using the equations proposed by Harris \& Benedict and the European Society for Clinical Nutrition and Metabolism recommendation. The nutritional status of the patients was determined by anthropometric assessment.

\section{Results}

High rates of malnutrition were found in both hospitals, especially when the mid-arm muscle area was used as the indicator of nutritional status. The energy prescription was adequate for $45.71 \%$ and $40.74 \%$ of the patients of hospitals 1 and 2, respectively.

\section{Conclusion}

Inadequate enteral feeding associated with high rates of malnutrition was found in both hospitals. The data obtained in this study show that both enteral feeding services need improvements. Standardization of enteral feeding practices and regular patient assessment are also needed.

Indexing terms: Nutritional status. Enteral nutrition. Nutrition therapy.

\section{N T R O D U ÇÃ O}

A utilização da Terapia Nutricional Enteral (TNE) tem demonstrado inúmeras vantagens para o paciente hospitalizado, como melhora na resposta imunológica ${ }^{1}$ diminuição de complicações clínicas ${ }^{2,3}$, assim como reduções de custos e do tempo de internação ${ }^{4}$. Mesmo com a freqüente utilização da TNE, a adequada prescrição de nutrientes por via enteral ainda é um grande desafio para os profissionais, pois existem dificuldades em determinar as necessidades nutricionais adequadas a cada indivíduo.

As conseqüências da incorreta administração nutricional, principalmente energética e protéica, podem agravar os quadros clínicos dos indivíduos. Oferta de energia insuficiente pode ser denominada como hipoalimentação ou underfeeding ${ }^{5}$, e suas conseqüências podem ser aumento no período de internação, comprometimento respiratório, demora na cicatrização de feridas, aumento do período de uso da ventilação mecânica, diminuição da integridade intestinal e também da resposta imunológica, assim como a desnutrição hospitalar ${ }^{6,7}$. Em contrapartida, uma oferta energética excessiva ou overfeeding tem sido mais presente nas terapias nutricionais ${ }^{6,8} \mathrm{e}$ pode acarretar efeitos prejudiciais como complicações metabólicas, aumento do gasto energético, comprometimento respiratório, disfunção hepática, aumento da morbimortalidade, estresse fisiológico, hiperglicemia e aumento da produção de dióxido de carbono ${ }^{6-8}$.

Para determinar as necessidades energéticas existem vários métodos. A calorimetria indireta $(\mathrm{Cl})$ é a mais indicada 5,8 , já que estima as necessidades energéticas por meio da medida da quantia de oxigênio consumido e de dióxido de carbono produzido ${ }^{9}$. Entretanto, a $\mathrm{Cl}$ possui limitações, como custo elevado dos equipamentos, treinamento da equipe e demanda de tempo para execução do procedimento ${ }^{10,11}$. Dessa forma, a maioria das instituições hospitalares ainda utiliza equações para determinar as quantidades energéticas diárias de cada paciente ${ }^{11,12}$.

Uma das equações mais utilizadas, devido à praticidade e facilidade de uso ${ }^{13}$, é a proposta por Harris \& Benedict ${ }^{14}$, segundo a qual se estima a energia necessária para cada indivíduo, utilizando o peso, a estatura, a idade e o sexo. Tem sido bastante utilizado também o valor de 25 a 
$35 \mathrm{kcal} / \mathrm{kg} / \mathrm{dia}$, preconizado pela Sociedade Espanhola de Nutrição Parenteral e Enteral $(\mathrm{SENPE})^{15}$ e de 20 a $30 \mathrm{kcal} / \mathrm{kg} / \mathrm{dia}$, recomendado recentemente pela European Society for Clinical Nutrition and Metabolism (ESPEN) ${ }^{16}$.

Os objetivos deste estudo foram avaliar o perfil nutricional e as necessidades energéticas de pacientes em uso da terapia nutricional enteral em dois hospitais da região metropolitana de Maringá (PR), Brasil.

\section{M É T O D O S}

Os pacientes foram incluídos na amostra após leitura e assinatura pelos próprios pacientes ou responsáveis, do Termo de Consentimento Livre e Esclarecido. Este estudo atendeu às Normas Regulamentares de Pesquisa envolvendo Seres Humanos - Resolução 196/96, do Conselho Nacional de Saúde, e foi aprovado pelo Comitê de Ética e Pesquisa da Universidade Estadual de Maringá (Registro número 203/2004).

Fizeram parte da amostra 62 pacientes em uso de terapia nutricional enteral tendo como critérios de inclusão: idade igual ou maior que 18 anos; atendimento pelo Sistema Único de Saúde (SUS) e em uso de nutrição enteral total (excluindo-se pacientes com nutrição oral e/ou parenteral). Pacientes com edema e/ou impossibilidade de aferição das medidas antropométricas foram excluídos da amostra.

A coleta de dados ocorreu em dois hospitais gerais da micro-região de Maringá (PR), qualificados abaixo:

Hospital 1: localizado no município de Maringá (PR), é um hospital escola e possui atendimento exclusivamente pelo SUS. Essa instituição de saúde tem cadastramento como Unidade de Assistência de Alta Complexidade em Terapia Nutricional no SUS, recebendo um valor diário por cada paciente em terapia de nutrição enteral, por isso, os pacientes recebem fórmulas enterais industrializadas e em sistema fechado.
Hospital 2: localizado no município de Sarandi (PR), e atende pacientes pelo SUS, conveniados e particulares. Este hospital utiliza uma fórmula de dieta industrializada enteral padrão para todos os pacientes do SUS e a infusão ocorre em sistema aberto. O hospital não possui o cadastramento no SUS para Terapia Nutricional.

Para analisar as necessidades energéticas, utilizou-se análise retrospectiva e o aporte energético foi avaliado em dois aspectos: energia prescrita e energia ingerida; ambos foram comparados à energia proposta para verificar a adequação energética.

Adequação energética: dois parâmetros foram adotados para avaliar a adequação de energia: a) equação proposta por Harris \& Benedict ${ }^{14}$, juntamente com fatores de lesão e atividade propostos Long et al. ${ }^{17}$. Esta equação foi escolhida pois é o método mais utilizado pelos dois hospitais para estimar energia; b) kcal/kg/ dia recomendados pela ESPEN (20 a 30kcal/peso ideal/dia). Esta segunda proposta foi adotada neste estudo por ser uma recomendação atualizada e adotada internacionalmente ${ }^{16}$.

Energia prescrita: os dados foram coletados nos prontuários dos pacientes, conforme as prescrições dietéticas e de soro glicosado.

Energia ingerida: os dados foram coletados nos prontuários dos pacientes conforme anotações da equipe de enfermagem para as dietas enterais e soro glicosado.

Para avaliar o perfil nutricional dos pacientes utilizou-se o Índice de Massa Corporal (IMC), a Prega Cutânea Tricipital (PCT) e a Área Muscular do Braço (AMB). Foram aplicados para os pacientes de 18 a 65 anos os parâmetros propostos por Frisancho ${ }^{18}$; os pontos de corte adotados classificaram os indivíduos como eutróficos (entre os percentis 15 a 75), desnutridos (abaixo do percentil 15) ou com excesso de peso (acima do percentil 75 para a PCT e AMB). Para avaliar o IMC utilizou-se a classificação da World Health Organization $^{19}$, sendo que aqueles com o IMC de 18,5 a $25 \mathrm{~kg} / \mathrm{m}^{2}$ foram considerados eutróficos, 
abaixo de $18,5 \mathrm{~kg} / \mathrm{m}^{2}$ desnutridos e acima de $25 \mathrm{~kg} / \mathrm{m}^{2}$ com excesso de peso. Para os pacientes acima de 65 anos foram adotados os parâmetros de Chumlea et al..$^{20}$, considerando-se eutróficos os pacientes que apresentaram IMC, PCT e AMB entre os percentis 5 a 95, desnutridos abaixo do percentil 5 e em excesso de peso acima do percentil 95.

Os dados foram analisados utilizando o Software Statistica 6.0. Análise estatística descritiva foi utilizada em cada variável. Diferenças entre os dados foram analisadas com o Teste $t$ Student e Qui-quadrado e os resultados foram considerados significantes quando $p<0,05$. Medidas para análise das relações entre as variáveis (dados antropométricos $x$ necessidades energéticas) foram calculadas pela Correlação de Pearson com o critério para análise do coeficiente (r), segundo Tritschler ${ }^{21}$.

\section{RES U L T A D OS}

Participaram da pesquisa 62 pacientes em uso da Terapia Nutricional Enteral, sendo 35 do Hospital 1 e 27 do Hospital 2. As características principais dos pacientes encontram-se na Tabela 1. Os pacientes avaliados são, na maioria, do sexo masculino, $71,43 \%$ e $66,67 \%$, nos hospitais 1 e

Tabela 1. Características de pacientes em uso de terapia nutricional enteral em dois hospitais da Região Metropolitana de Maringá (PR), 2006.

\begin{tabular}{|c|c|c|c|c|}
\hline \multirow{2}{*}{$\begin{array}{l}\text { Características dos pacientes } \\
\text { Idade (anos)* }\end{array}$} & \multicolumn{2}{|c|}{ Hospital $1(n=35)$} & \multicolumn{2}{|c|}{ Hospital $2(n=27)$} \\
\hline & M & DP & M & DP \\
\hline & 52,88 & 18,85 & 54,22 & 21,86 \\
\hline Mínimo & \multicolumn{2}{|c|}{20} & \multicolumn{2}{|c|}{20} \\
\hline \multirow[t]{2}{*}{ Máximo } & \multicolumn{2}{|c|}{86} & \multicolumn{2}{|c|}{89} \\
\hline & $n$ & $\%$ & $n$ & $\%$ \\
\hline \multicolumn{5}{|l|}{$\operatorname{SexO}{ }^{*}$} \\
\hline Masculino & 25 & 71,43 & 18 & 66,67 \\
\hline Feminino & 10 & 28,57 & 9 & 33,33 \\
\hline \multicolumn{5}{|l|}{ Clínicas } \\
\hline Unidade de Terapia Intensiva (UTI) & 28 & 80,00 & 21 & 77,78 \\
\hline Médicas & - & & 6 & 22,22 \\
\hline Cirúrgicas & 7 & 20,00 & - & \\
\hline \multicolumn{5}{|l|}{ Morbidades (motivo da internação) } \\
\hline Acidente vascular cerebral & 10 & 28,57 & 10 & 37,04 \\
\hline Traumatismo craniano & 3 & 8,57 & 7 & 25,93 \\
\hline Traumas & 5 & 14,28 & 4 & 14,81 \\
\hline Insuficiência respiratória & 5 & 14,28 & 3 & 11,11 \\
\hline Queimaduras & 2 & 5,71 & - & \\
\hline Insuficiência cardíaca congestiva & - & & 1 & 3,70 \\
\hline Insuficiência renal crônica & 3 & 8,57 & - & \\
\hline Cirrose hepática & 1 & 2,86 & - & \\
\hline Pancreatite crônica & 2 & 2,86 & 1 & 3,70 \\
\hline Doença inflamatória intestinal & 3 & 2,86 & - & \\
\hline Diabetes Mellitus & 4 & 2,86 & - & \\
\hline Hipertensão arterial sistêmica & 5 & 2,86 & 1 & 3,70 \\
\hline Câncer & 2 & 5,71 & - & \\
\hline \multicolumn{5}{|l|}{ Alta* } \\
\hline Nutrição enteral & 8 & 22,86 & 9 & 33,33 \\
\hline Nutrição oral & 16 & 45,71 & 7 & 25,92 \\
\hline Óbito & 11 & 31,43 & 11 & 40,74 \\
\hline
\end{tabular}

* Sem diferença significante entre os hospitais para $p>0,05$ (Teste $t$ Student e Qui-quadrado).

M: média; DP: desvio-padrão. 
2 , respectivamente. A idade média foi semelhante em ambos os hospitais ( $p>0,05)$, sendo 52,88 anos (Desvio padrão - $D P=18,85)$ e 54,22 anos ( $D P=21,86)$, nos hospitais 1 e 2 , respectivamente.

Com relação às clínicas em que os indivíduos estavam internados, pode-se verificar que no Hospital 1, 80\% dos pacientes estavam na Unidade de Terapia Intensiva (UTI) e 20,00\% na Clínica Cirúrgica, enquanto no Hospital 2, 77,78\% dos pacientes estavam na UTI e 22,22\% na Clínica Médica.

Pelo fato de os hospitais em estudo serem do tipo geral, as causas de internações foram variadas, encontrando-se diagnósticos com o maior percentual em Acidente Vascular Cerebral (AVC) em ambos os hospitais, seguido de Traumatismo Craniano no Hospital 2 e Traumas e de Insuficiência Respiratória no Hospital 1 (Tabela 1).

Avaliou-se também o tipo de terapia nutricional utilizado pelos pacientes no momento da alta hospitalar. Pode-se observar que dos avaliados no Hospital 1, $16(45,71 \%)$ pacientes tiveram alta hospitalar com alimentação por via oral, 8 (22,86\%) continuaram com nutrição enteral e 11 (31,43\%) pacientes foram a óbito. Dos 27 pacientes do Hospital 2, 9 (33,33\%) tiveram alta com nutrição enteral, 7 (25,92\%) com nutrição oral e 11 (40,74\%) também foram a óbito. Por meio do teste Qui-quadrado detecta-se que não houve diferenças significantes entre essas variáveis $(p>0,05)$.

Não houve diferenças significantes entre os hospitais para as medidas de peso, estatura, IMC, PCT e AMB. Com relação ao estado nutricional, o IMC médio dos pacientes no Hospital 1 foi igual a $20,15 \mathrm{~kg} / \mathrm{m}^{2}(\mathrm{DP}=4,18)$ e no Hospital 2, $19,12 \mathrm{~kg} / \mathrm{m}^{2} \quad(\mathrm{DP}=4,95)$. Índices de desnutrição ocorreram em ambos os hospitais, $34,28 \%$ no Hospital 1 e 44,44\% no Hospital 2 (Figura 1). Ao analisar a PCT, os percentuais de déficit de massa gorda foram de $25,71 \%$ e $33,33 \%$ nos hospitais 1 e 2, respectivamente. As maiores proporções de desnutrição foram encontradas na análise da $\mathrm{AMB}$, demonstrando redução de massa magra em $54,28 \%$ nos pacientes do Hospital 1 e 59,26\% nos do Hospital 2. Em todas as variáveis não foram encontradas diferenças significantes, sendo $p>0,05$.

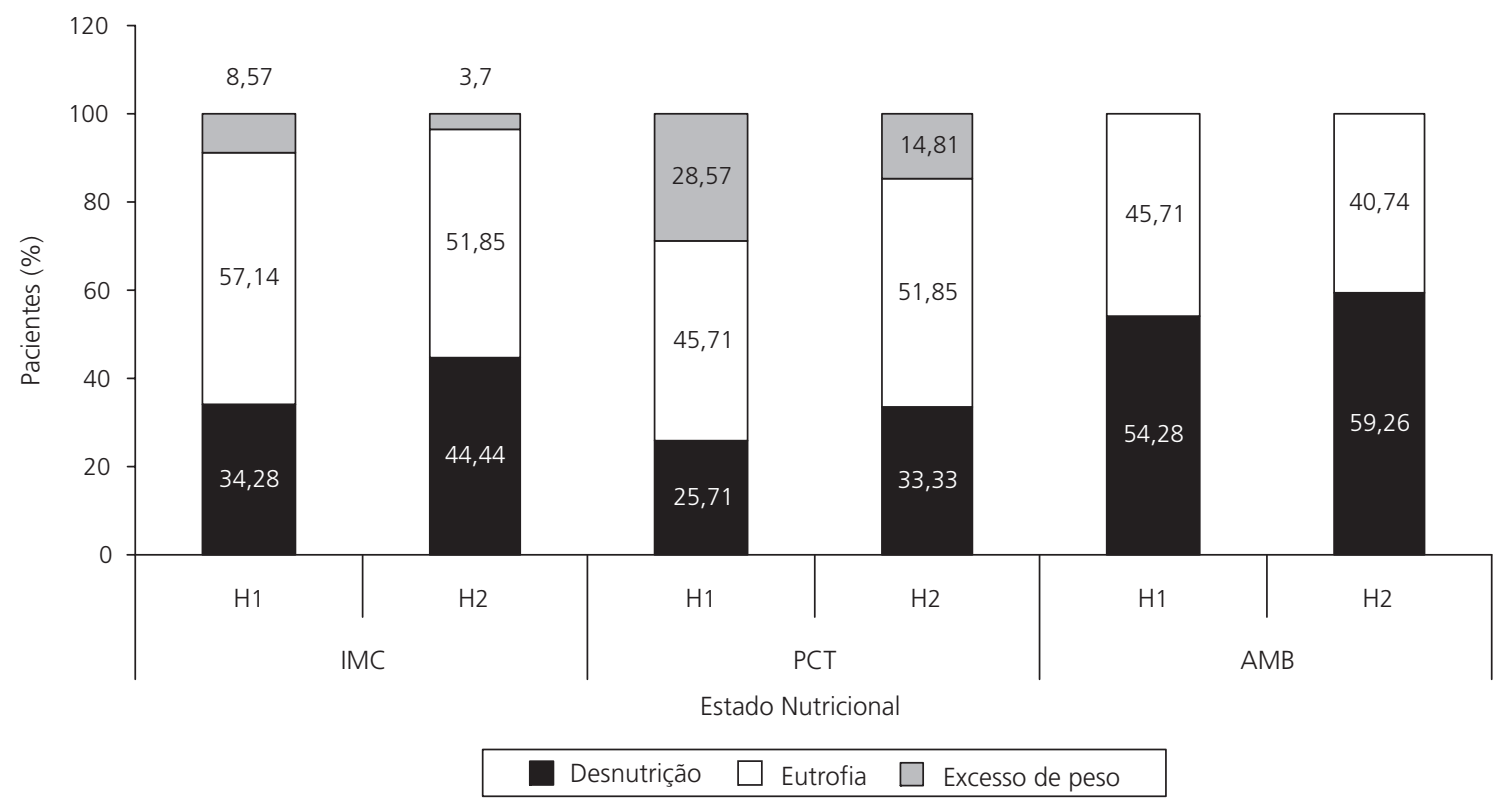

Figura 1. Distribuição percentual de pacientes em uso de terapia nutricional enteral quanto ao estado nutricional em dois hospitais da Região Metropolitana de Maringá (PR), 2006.

IMC: índice de massa corporal; PCT: prega cutânea tricipital; AMB: área muscular do braço; H1: hospital 1; H2: hospital 2. 
Na Figura 2 está representada a freqüência de pacientes que tiveram as prescrições classificadas como adequadas ou não, utilizando-se como parâmetro as equações de Harris \& Benedict $^{14}$. Pode-se observar que todas as prescrições em ambos os hospitais estavam inadequadas, sendo que no Hospital $180 \%$ e no Hospital $281,48 \%$ dos pacientes tiveram as prescrições abaixo da recomendação e 20\% (H1) e 18,52\% $(\mathrm{H} 2)$ estavam acima das recomendações. Se as prescrições fossem baseadas neste método, as mesmas atingiriam a média de $38,44(D P=6,30)$ no H1 e $36,98 \mathrm{kcal} / \mathrm{kg} / \mathrm{dia}(\mathrm{DP}=3,51)$ no H2, estando acima das recomendações da ESPEN de 20 a $30 \mathrm{kcal} / \mathrm{kg} / \mathrm{dia}$.

Na Figura 3 pode-se analisar o total energético prescrito tomando-se como parâmetro 20 a $30 \mathrm{kcal} / \mathrm{kg} / \mathrm{dia}$ recomendado pela ESPEN. Verifica-se que as prescrições estavam adequadas em $45,71 \%(\mathrm{H} 1)$ e $40,74 \%$ ( $\mathrm{H} 2$ ) e ainda existe um percentual elevado de prescrições energéticas acima da recomendação, ou seja, 45,71\% (H1) e $44,44 \%$ (H2). No Hospital 1, 8,57\% dos pacientes tiveram as prescrições abaixo da recomendação, enquanto no Hospital 2, $14,81 \%$ dos pacientes enquadraram-se nesta situação. Os valores médios em relação à prescrição energética no Hospital 1 e no Hospital 2 foram semelhantes $(p>0,05)$.

Na Figura 2 verifica-se que nos dois hospitais $100 \%$ dos avaliados tiveram a ingestão de energia abaixo das recomendações em relação à estimativa baseada em Harris \& Benedict ${ }^{14}$. Entretanto, quando se analisa a ingestão com o parâmetro de 20 a $30 \mathrm{Kcal} / \mathrm{kg} / \mathrm{dia}$, pode-se constatar que esse percentual cai para $54,29 \%(\mathrm{H} 1)$ e $44,44 \%$ (H2). Pode-se observar ainda que $42,85 \%$ dos pacientes no Hospital 1 tiveram consumo adequado e $2,85 \%$ acima das recomendações. No Hospital $244,44 \%$ estavam adequados e 11,11\% acima de $30 \mathrm{kcal} / \mathrm{kg} / \mathrm{dia}$.

Na Figura 3 pode-se observar que $71,43 \%$ dos pacientes do $\mathrm{H} 1$ e $59,26 \%$ dos pacientes do H2 não consumiram o valor energético prescrito em prontuário. Dessa forma pode-se verificar que no Hospital 2, a real ingestão de energia foi melhor que no Hospital 1.

Ao aplicar a Matriz de Correlação de Pearson, verifica-se que a densidade energética ingerida no Hospital 1 teve correlação moderada com o IMC e os pesos dos pacientes. No Hospital 2 houve correlações fracas entre a energia ingerida e as variáveis: período de internação, IMC, $\mathrm{AMB}$ e peso.

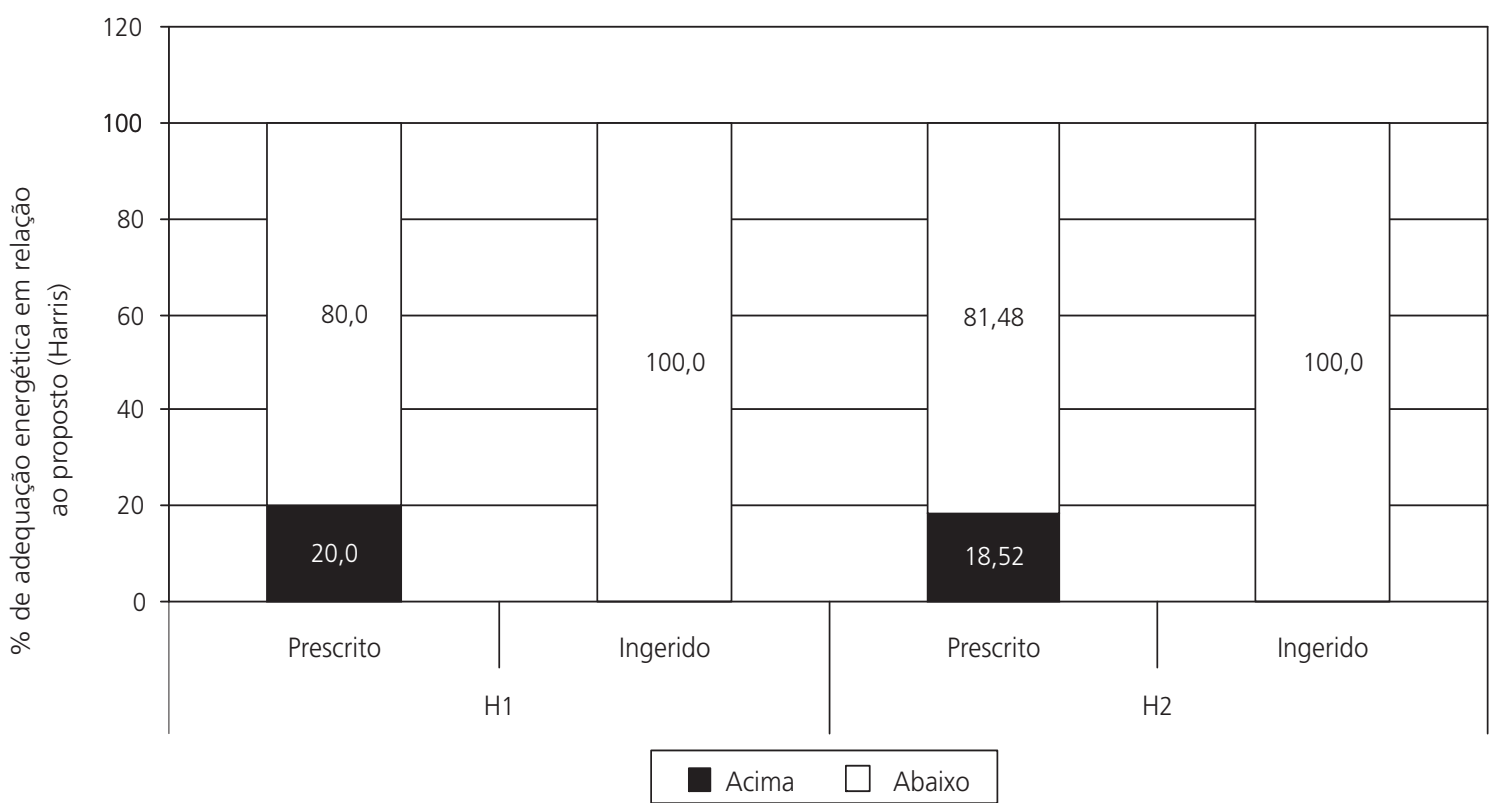

Figura 2. Distribuição percentual de pacientes em uso de terapia nutricional enteral, quanto à adequação de energia proposta por Harris \& Benedict ${ }^{14}$ em dois hospitais da Região Metropolitana de Maringá (PR), 2006.

H1: hospital 1 e H2: hospital 2. 


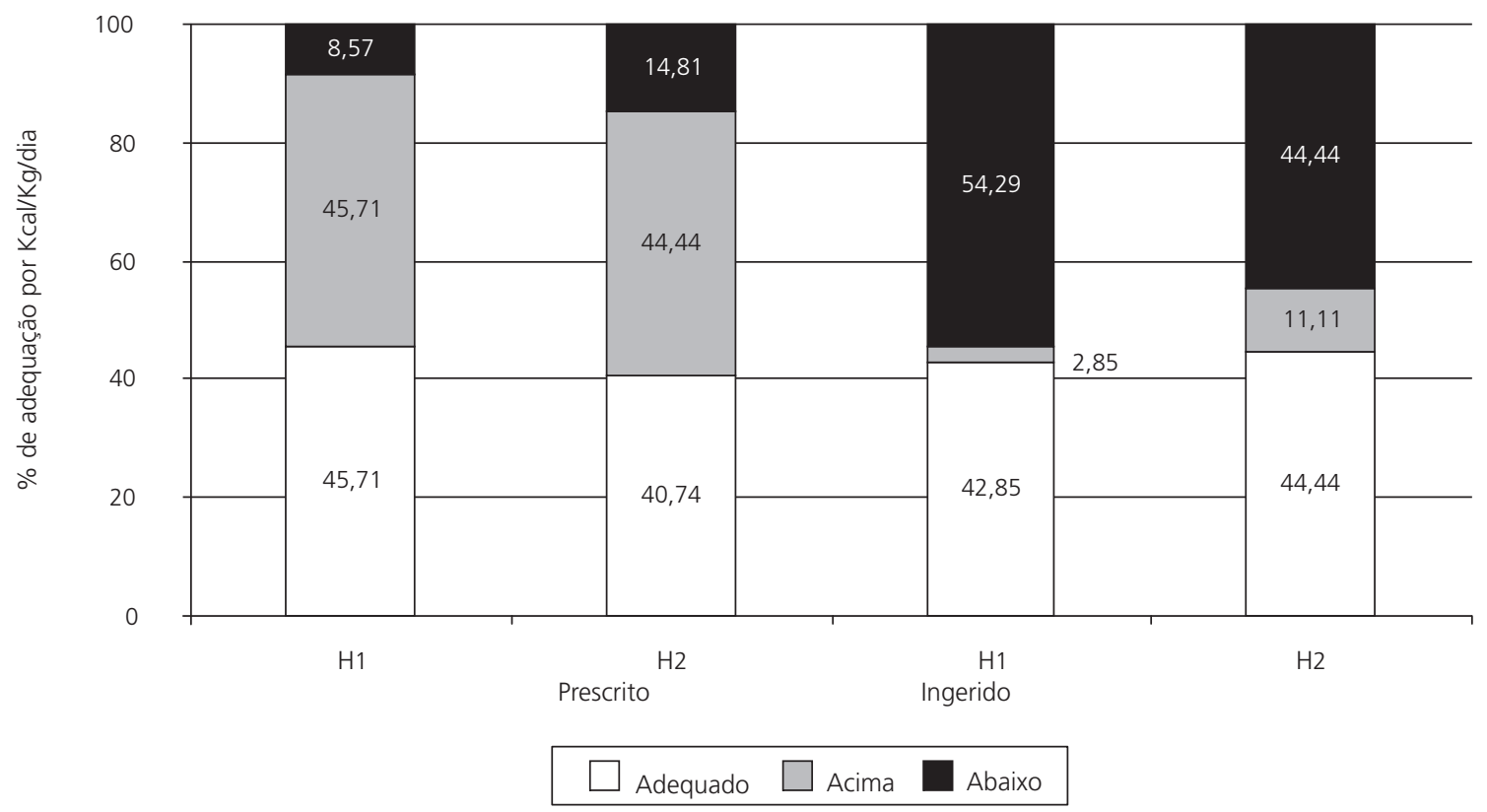

Figura 3. Distribuição da recomendação energética a partir de 20 a 30kcal $/ \mathrm{kg} / \mathrm{dia}$ no suporte nutricional de pacientes em uso de terapia nutricional enteral quanto a calorias prescritas e ingeridas em dois hospitais da Região Metropolitana de Maringá (PR), 2006.

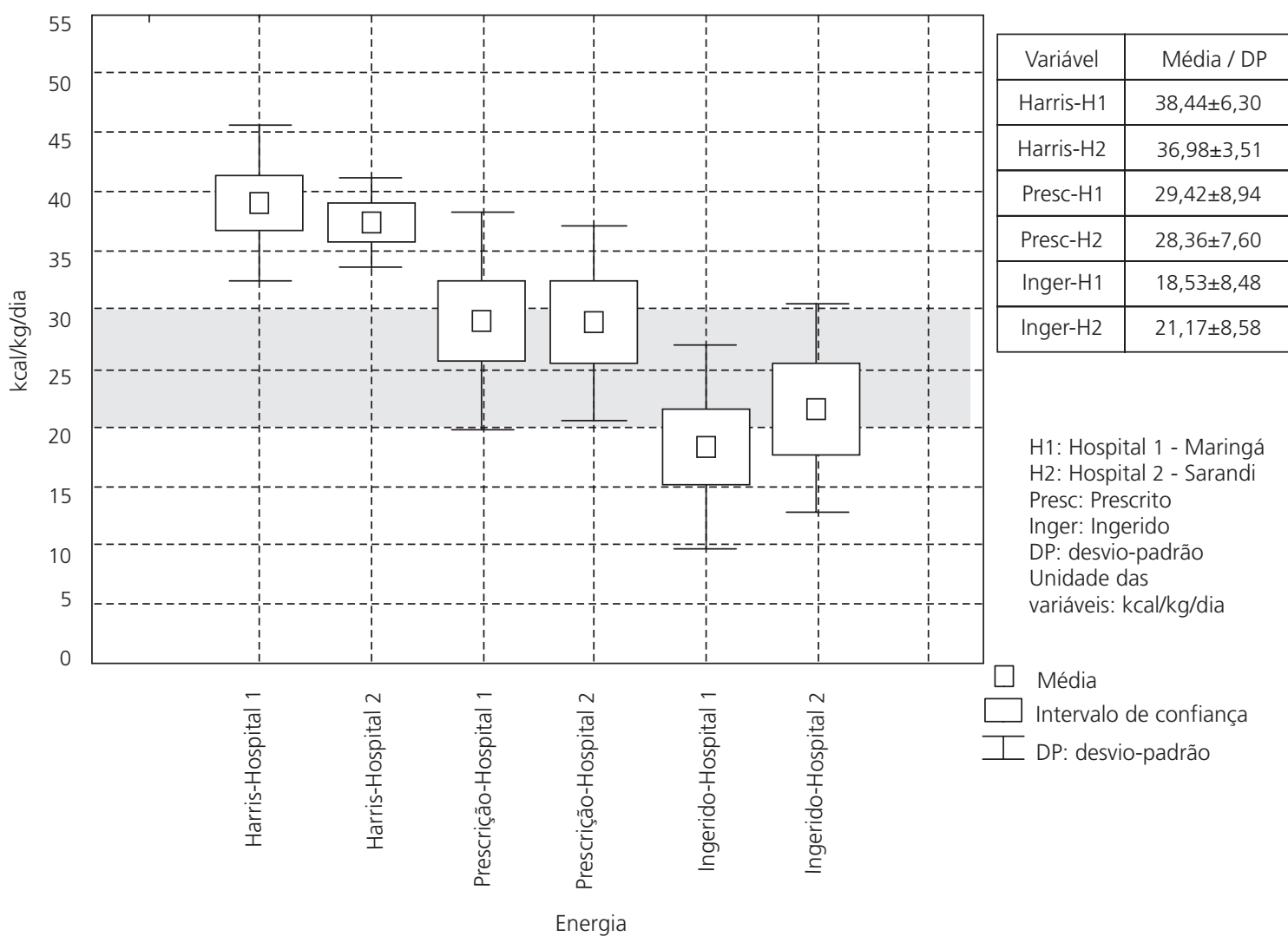

Figura 4. Distribuição das recomendações de energia ( $\mathrm{kcal} / \mathrm{kg} / \mathrm{dia}$ ) para pacientes em uso de terapia nutricional enteral em dois hospitais da Região Metropolitana de Maringá (PR), 2006.

Nota: Cada valor energético no gráfico está expresso em média e desvio-padrão, sendo considerado adequado entre 20 a $30 \mathrm{kcal} / \mathrm{kg} / \mathrm{dia}$ (faixa cinza) 
As variáveis Energia Proposta por Harris \& Benedict ${ }^{14}$; Energia Prescrita e Energia Consumida foram comparadas com os valores sugeridos pela ESPEN (Figura 4). Pode-se observar que as recomendações de energia propostas por Harris \& Benedict estão acima de $30 \mathrm{kcal} / \mathrm{kg} / \mathrm{dia}$, como citado anteriormente. Os valores energéticos prescritos são suficientes para fornecer entre 29,42 $(\mathrm{DP}=8,94)$ no Hospital 1 e $28,36 \mathrm{kcal} / \mathrm{kg} / \mathrm{dia}$ $(\mathrm{DP}=7,60)$ no Hospital 2 e, mesmo as médias estando de acordo com as recomendações (faixa cinza), observa-se que existem pacientes acima do preconizado. Esse fato também foi constatado na Figura 3.

Com relação à ingestão verifica-se que no Hospital 1 a média de ingestão de energia foi menor $(18,53, D P=8,48 \mathrm{kcal} / \mathrm{kg} / \mathrm{dia})$ que no Hospital 2 21,17 (DP=8,58) kcal/kg/dia. Entretanto, essa diferença não foi significante $(p>0,05)$.

Ao comparar as variáveis, pode-se verificar que a Energia Proposta por Harris é maior do que a Energia Prescrita e que esta é maior do que a Energia Ingerida e que existem diferenças significantes $(p<0,05)$ entre esses valores tanto no Hospital 1 como no Hospital 2.

\section{I S C U S S Ã O}

Pôde-se encontrar uma proporção elevada de desnutrição nos pacientes avaliados, com valores decrescentes para AMB, IMC e PCT. Sabe-se que na desnutrição energética protéica ocorre a depleção de massa magra, e em segundo plano, da gordura corporal ${ }^{22}$. Talvez esta seja a hipótese para que os pacientes do presente estudo tenham apresentado maior deficiência de massa magra.

A desnutrição também foi encontrada em outras pesquisas. Silva et al..$^{23}$ encontraram $55,9 \%$ de desnutrição nos pacientes em terapia de nutrição enteral. Em estudo realizado no Brasil com 4 mil pacientes, Waitzberg et al. ${ }^{3}$ identificaram que 6,1 \% faziam uso de nutrição enteral, e que destes, $10,1 \%$ estavam em desnutrição. Em estudo semeIhante, realizado na América Latina por Correia \& Campos $^{24}, 6,3 \%$ dos pacientes estavam em uso de NE e destes $5,6 \%$ foram desnutridos.
A análise energética utilizando-se como parâmetro o cálculo por meio de Harris \& Benedict, demonstrou que a prescrição e a ingestão de energia por parte dos pacientes estavam inadequadas nos dois hospitais. De Jonghe et al. ${ }^{25}$ utilizaram também a equação de Harris \& Benedict ${ }^{14}$ e o fator estresse de Long et al. ${ }^{17} \mathrm{e}$ detectaram que mais da metade dos pacientes ingeriram menos que $70 \%$ das necessidades energéticas propostas. O'Leary-Kelley et al. ${ }^{26}$ detectaram que, mais da metade dos pacientes receberam menos que $50 \%$ das necessidades energéticas.

A inadequação energética constatada por meio deste método de cálculo das necessidades é preocupante, já que os profissionais nos dois serviços relatam que quando utilizam algum método para estimar as necessidades de energia, este é o cálculo por Harris \& Benedict e por isso é essencial que exista melhor adequação quando estudado por estas equações.

Ao analisar com o padrão de 20 a 30kcal/ $\mathrm{kg} / \mathrm{dia}$, a adequação se modifica e a energia prescrita e ingerida fica acima de $40 \%$ da adequação em ambos os hospitais, entretanto ainda existiu déficit do consumo energético em, aproximadamente, metade da população estudada. O'Leary-Kelley et al. ${ }^{26}$ encontraram $68 \%$ de inadequação do consumo energético e Roberts et al. ${ }^{27}$ verificaram que cerca de $60 \%$ dos pacientes receberam menos que $85 \%$ das necessidades.

No entanto, é preciso considerar que Harris \& Benedict superestimam as necessidades energéticas $^{15}$. Em estudo realizado por Kyle et al.28, utilizando essas equações em comparação à Calorimetria Indireta, foi detectado que para pacientes em ventilação mecânica, a aplicação da equação de Harris \& Benedict superestimou em $119,00 \%$ as necessidades energéticas e para pacientes sem ventilação mecânica ocorreu superestimação da ordem de 123,00\%. Esse fato também ficou demonstrado neste estudo, já que os pacientes tiveram a recomendação proposta por Harris acima de $30 \mathrm{kcal} / \mathrm{kg} / \mathrm{dia}$ em 128,13\% (Hospital 1) e em 123,26\% (Hospital 2).

Diferentes métodos para o cálculo das necessidades energéticas são utilizados em pesqui- 
sas, pode-se citar a utilização da calorimetria indireta como sendo o padrão ouro. Pesquisadores como McClave et al..$^{5}$ Engel et al. ${ }^{2}$ utilizaram $\mathrm{Cl}$ em seus trabalhos. As equações propostas por Harris \& Benedict ${ }^{14}$ são do ano de 1919, mas ainda continuam sendo utilizadas, como nas pesquisas de Ravasco \& Camilo8, O'Leary-Kelley et al. ${ }^{26} \mathrm{e}$ Kyle et al. ${ }^{28}$. O cálculo sugerido pela ESPEN são utilizados e encontrados na metodologia de pesquisas como, por exemplo: Woien \& Bjork ${ }^{9}$, Robert et al. ${ }^{27}$, Petros \& Engelmann ${ }^{29}$ e possuem a vantagem de maior praticidade de uso, já que utiliza apenas o peso multiplicado por 20 a 30kcal/dia.

Apesar de os serviços oferecidos pelos hospitais serem diferentes quanto ao tipo de dietas ofertadas (o Hospital 1 oferecia dieta industrializada e de acordo com a doença, já o Hospital 2 possuía apenas uma dieta padrão industrializada, independentemente da morbidade), essa particularidade dos hospitais não influenciou a análise das necessidades energéticas, já que as diferenças não foram significantes entre os mesmos. Sabe-se que energia é amplamente estudados por diversos autores $16,7,25,27$, entretanto na análise de adequação de dietas enterais, outros fatores devem ser considerados. Pode-se citar como alguns exemplos, o conteúdo em fibras para o tratamento de indivíduos com doenças intestinais, a presença de antioxidantes para melhora da resposta imune nos pacientes graves, assim como a presença de imunoestimulantes (arginina e glutamina), principalmente no uso de pacientes cirúrgicos. Portanto, é necessário investigar outros nutrientes para detectar possíveis diferenças significantes entre os serviços estudados.

Foram detectadas diferenças significantes entre as prescrições e a oferta de energia nos dois serviços. Outros trabalhos também verificaram discrepâncias entre a prescrição e o consumo, como por exemplo: De Jonghe et al. ${ }^{25}$ encontraram que dos valores energéticos prescritos apenas $71 \%$ foram ofertados. Resultados semelhantes foram encontrados em outros estudos ${ }^{10,29,30}$. As causas para a diferença entre ingestão e prescrição não foram investigadas nesta pesquisa, entretanto outros autores demonstraram que as causas mais prováveis para essa diferença foram intolerância digestiva ${ }^{25}$, diarréia, vômito e distensão abdominal27,29.

\section{O N CLUS Ã O}

As necessidades energéticas estimadas por meio de Harris \& Benedict são maiores do que 20 a $30 \mathrm{kcal} / \mathrm{kg} / \mathrm{dia}$ recomendadas pela European Society for Clinical Nutrition and Metabolism (ESPEN), havendo com seu uso risco de hiperalimentação ou overfeeding. Entretanto, verificou-se que em ambos hospitais estudados, as prescrições energéticas ainda estão inadequadas em mais da metade dos avaliados (acima e abaixo da recomendação), mesmo utilizando-se as recomendações de 20 a $30 \mathrm{kcal} / \mathrm{kg} / \mathrm{dia}$. Quanto à ingestão, essa também estava deficiente em, aproximadamente, $40 \%$ nos dois serviços. Os dados obtidos mostram que há indicação de hipoalimentação ou underfeeding, sendo reforçados pelos resultados de desnutrição (em maior índice para a área muscular do braço), mesmo com a utilização da terapia de nutrição enteral; mostram também a necessidade de melhoras nos dois serviços de nutrição enteral, havendo necessidade de adoção de medidas de padronização, avaliação periódica dos pacientes e maior integração dos profissionais envolvidos.

\section{COLABORADORES}

V.T. NOZAKI e R.M. PERALTA participaram igualmente da concepção da pesquisa, da revisão da literatura, da coleta dos dados, da análise das variáveis e da redação do artigo.

\section{REFERÊ NCIAS}

1. Genton L, Hans D, Kyle UG, Pichard C. Dual-energy X-ray absorptiometry and body composition: differences between devices and comparison with reference methods. Nutrition. 2002; 18(1):66-70.

2. Engel JM, Muhling J, Junger $A$, Menges $T$, Karcher B, Hempelmann G. Enteral nutrition practice in a surgical intensive care unit: what proportion of energy expenditure is delivered enterally? Clin Nutr. 2003; 22(2):187-92. 
3. Waitzberg DL, Caiafa WT, Correia MITD. Hospital malnutrition: the Brazil National Survey (IBRANUTRI): a study of 4000 patients. Nutrition. 2001; 17(7): 573-80.

4. Watanabe S, Cukier C, Magnoni D, Guimarães RN, Urenhiuki KL, Rauba A. Nutrição enteral precoce reduz tempo de internação hospitalar e melhora reembolso diário do Sistema Único de Saúde (SUS) ao hospital. Rev Bras Nutr Clin. 2002; 17(2):47-50.

5. McClave AS, McClain CJ, Snider HL. Should indirect calorimetry be used as part of nutritional assessment? J Clin Gastroenterol. 2001; 33(1):14-9.

6. Krishnan JA, Parce PB, Matinez A, Diette GB, Brower RG. Caloric intake in medical ICU patients. Chest. 2003; 124(1):297-305.

7. Reid C. Frequency of under- and over feeding in mechanically ventilated ICU patients: causes and possible consequences. J Hum Nutr Diet. 2006; 19(1):13-22.

8. Ravasco P, Camilo ME. The impact of fluid therapy on nutrient delivery: a prospective evaluation of practice in respiratory intensive care. Clin Nutr. 2003; 22(1):87-92.

9. Woien H, Bjork IT. Nutrition of the critically ill patient and effects of implementing a nutritional support algorithm in ICU. J Clin Nurs. 2006; 15(2):168-77.

10. Klein CJ, Stanek GS, Wiles CE. Over feeding macronutrients to critically ill adults: metabolic complications. J Am Diet Assoc. 1998; 98(7):795-806.

11. Ireton-Jones C, Jones J. Should predictive equations or indirect calorimetry be used to design nutrition support regimens? NCP. 1998; 13(3):141-3.

12. MacDonald A, Hildebrandt L. Comparison of formulaic equations to determine energy expenditure in the critically III patient. Nutrition. 2003; 19(3):233-9.

13. Genton L, Dupertuis YM, J Romand, Simonet ML, Jolliet $\mathrm{P}$, Huber $\mathrm{O}$, et al. Higher calorie prescription improves nutrient delivery during the first 5 days of enteral nutrition. Clin Nutr. 2004; 23 (3): 307-15.

14. Harris JA, Benedict FG. A biometric study of basal metabolism in man. Washington (DC): Carnegie Institute of Washington; 1919. Publication n.279.

15. Sociedad Española de Nutrición Parenteral y Enteral: protocolos para la prescripción de nutrición parenteral y enteral. Documento 2-C-EP, 1998. Zaragoza: SENPE; 1998.

16. Kreymann KG, Berger MM, Deutz NEP, Hiesmayr M, Jolliete P, Kazandjiev G, et al. ESPEN Guidelines on Enteral Nutrition: intensive care. Clin Nutr. 2006; 25(2):210-23.

17. Long CL, Schaffel N, Geiger JW, Schiller WR, Blackmore WS. Metabolic response to injury and illness. Estimation of energy and protein needs from indirect calorimetry and nitrogen balance. J Pen. 1979; 3(6):452-6.

18. Frisancho AR. Anthropometric standards for assessment of growth and nutrition status. Michigan: The University of Michigan Press; 1990.

19. World Health Organization. Obesity: preventing and managing the global epidemic. Geneva: WHO; 1997. Report of WHO Consulation on Obesity.

20. Chumlea WC, Roche AF, Mukherjee D. Ross Laboratories. Nutritional assessment of the elderly through anthropometry. Ohio: Ross Laboratories; 1987.

21. Tritschler KA. Medida e avaliação em educação física e esportes. 5a. ed. São Paulo: Manole; 2003.

22. Waitzberg DL, Gama-Rodrigues J, Correia MITD. Desnutrição hospitalar no Brasil. In: Waitzberg DL, editor. Nutrição oral, enteral e parenteral na prática clínica. São Paulo: Atheneu; 2001. p.385-97.

23. Silva AFF, Campos DJ, Souza MH, Shieferdecker ME. Capacidade da terapia nutricional enteral em fornecer as necessidades calórico-protéicas de pacientes hospitalizados. Rev Bras Nutr Clin. 2003; 18(3):113-8.

24. Correia MITD, Campos ACL. Prevalence of Hospital malnutrition in Latin America: the Multicenter ELAN Study. Nutrition. 2003; 19(10):823-5.

25. De Jonghe B, Appere-De-Vechi C, Fournier M, Tran B, Merrer J, Melchior JC, et al. A prospective survey of nutritional support practices in intensive care unit patients: what is prescribed? What is delivered? Crit Care Med. 2001; 29(1): 8-12.

26. O'Leary-Kelley CM, Puntillo KA, Barr J, Sttots N, Douglas MK. Nutritional adequacy in patients receiving mechanical ventilation who are fed enterally. Am J Crit Care. 2005; 14(3):222-31.

27. Roberts SR, Kennerly DA, Keane D, George C. Nutrition support in the intensive care unit: adequacy, timeliness and outcomes. Crit Care Nurse. 2003; 23(6):49-57.

28. Kyle UG, Genton L, Heidegger CP, Maisonneuve N, Karsegard VL, Huber O, et al. Hospitalized mechanically ventilated patients are at higher risk of enteral underfeeding than non-ventilated patients. Clin Nutr. 2006; 25(5):727-35.

29. Petros S, Engelmann L. Enteral nutrition delivery and energy expenditure in medical intensive care patients. Clin Nutr. 2006; 25(1):51-9.

30. Couto JCF, Bento A, Couto CMF, Silva BCO, Oliveira IAG. Nutrição enteral em Terapia Intensiva: o paciente recebe o que prescrevemos? Rev Bras Nutr Clin. 2002; 17(2):43-6.

Recebido em: 24/8/2007

Versão final reapresentada em: 12/5/2008 Aprovado em: 30/3/2009 